\section{New Exponential Estimates for Time-Delay Systems}

\author{
Shengyuan $\mathrm{Xu}$, James Lam, and Maiying Zhong
}

\begin{abstract}
This note considers the problem of exponential stability for time-delay systems. In terms of linear matrix inequalities, a new sufficient condition for exponential stability is obtained. Based on this, an improved upper bound of the decay rate can be easily calculated. When time-varying norm-bounded parameter uncertainties appear, a new sufficient condition for robust exponential stability of uncertain time-delay systems is also provided. The reduced conservatism of the proposed conditions is illustrated via two numerical examples.
\end{abstract}

Index Terms-Exponential stability, linear matrix inequality (LMI), time-delay systems, uncertain systems.

\section{INTRODUCTION}

Time-delay systems have been investigated by many researchers since they are encountered in engineering systems, biology, economics, and other areas [7], [11]. Stability analysis of time-delay systems is of both practical and theoretical importance since time delays are frequently the main cause of instability and poor performance of a system. A great number of stability results have been proposed in the literature; see, e.g., [1]-[4], [6], [14]-[16], and the references therein. These results can be classified into two types according to their dependence of the delay size; that is, delay-dependent stability results and delay-independent ones. Delay-dependent stability results are generally less conservative than delay-independent ones.

It is noted that many stability results for time-delay systems are concerned with asymptotic stability. In practical applications, however, it is also important to find estimates of the transient decay rate of a delay system. Therefore, the problem of exponential stability has been studied. For instance, an estimate of the decay rate of a linear stable delay systems was given in [10], which was further improved in [5]. By using the properties of matrix measure, sufficient conditions for the exponential stability of time-delay systems were obtained in [13]. When time-varying delays appear, some robust exponential stability results were proposed in [12]. However, the conditions in both [12] and [13] are not easy to check. Very recently, by a linear matrix inequality (LMI) approach, exponential stability conditions were presented in [8] and [9], respectively. These conditions can be easily checked.

In this note, we provide a new exponential stability condition for time-delay systems by choosing an appropriate Lyapunov-Krasovskii functional and introducing slack variables. Based on this, an upper bound of the decay rate can be easily calculated. When time-varying norm-bounded parameter uncertainties appear, a robust exponential stability condition is also provided. Both the exponential stability and the robust exponential stability conditions are given in terms of LMIs. The proposed conditions in this note are less conservative than some

Manuscript received June 9, 2005; revised October 24, 2005. Recommended by Associate Editor S. Tarbouriech. This work was supported in part by RGC HKU 7028/04P, the Program for New Century Excellent Talents in University under Grant NCET-04-0508, the National Natural Science Foundation of P.R. China under Grant 60304001, and the Foundation for the Author of National Excellent Doctoral Dissertation of P.R. China under Grant 200240.

$\mathrm{S}$. Xu is with the Department of Automation, Nanjing University of Science and Technology, Nanjing 210094, P. R. China (e-mail: syxu02@yahoo.com.cn).

J. Lam is with the Department of Mechanical Engineering, University of Hong Kong, Hong Kong.

M. Zhong is with the Control Science and Engineering School, Shandong University, 250061 Jinan, P. R. China.

Digital Object Identifier 10.1109/TAC.2006.880783 of those in the literature, which is demonstrated via two numerical examples.

Notation: Throughout this note, for real symmetric matrices $X$ and $Y$, the notation $X \geq Y$ (respectively, $X>Y$ ) means that the matrix $X-Y$ is positive semidefinite (respectively, positive definite). $I$ is an identity matrix with appropriate dimension. The superscript " $T$ " represents the transpose. The notations $|\cdot|$ and $\|\cdot\|$ refer to the Euclidean vector norm and the induced matrix two-norm, respectively. We use $\lambda_{\min }(\cdot)$ and $\lambda_{\max }(\cdot)$ to denote the minimum and maximum eigenvalue of a symmetric matrix, respectively. Matrices, if their dimensions are not explicitly stated, are assumed to have compatible dimensions.

\section{MAIN RESULTS}

Consider the following time-delay system:

$$
\begin{aligned}
(\Sigma): \quad \dot{x}(t) & =A x(t)+A_{1} x(t-h) \\
x(t) & =\varphi(t) \quad \forall t \in[-h, 0]
\end{aligned}
$$

where $x(t) \in \mathbb{R}^{n}$ is the state, and $\varphi(t)$ is the initial condition. The scalar $h>0$ is the delay of the system, $A$ and $A_{1}$ are known real constant matrices.

Definition 1: System $(\Sigma)$ is said to be exponentially stable with a decay rate $\lambda$ if there exist scalars $\sigma \geq 1$ and $\lambda>0$ such that $|x(t)| \leq$ $\sigma e^{-\lambda t}|\varphi|_{h}$ where $|\varphi|_{h}=\sup _{-h<\theta<0}|\varphi(\theta)|$.

We provide a new exponential stability test for delay system $(\Sigma)$ in the following theorem.

Theorem 1: For given scalars $\lambda>0$ and $h>0$, the time-delay system $(\Sigma)$ is exponentially stable with a decay rate $\lambda$ if there exist matrices $P_{1}>0, P_{3}>0, Q>0, Z_{1}>0, Z_{2}>0, Y, W, S$, and $P_{2}$ such that the LMIs, as shown in (3) and (4) at the bottom of the next page, hold.

$$
\begin{aligned}
\tilde{A}(\lambda)= & A+\lambda I \\
\tilde{A}_{1}(\lambda)= & e^{\lambda h} A_{1} \\
\Omega(\lambda)= & P_{1} \tilde{A}(\lambda)+\tilde{A}(\lambda)^{T} P_{1}+P_{2} \\
& +P_{2}^{T}-Y-Y^{T}+Q+h Z_{2} \\
\Psi_{1}(\lambda)= & P_{1} \tilde{A}_{1}(\lambda)-P_{2}+Y-W^{T} \\
\Psi_{2}(\lambda)= & \tilde{A}(\lambda)^{T} P_{2}+P_{3}-S^{T} \\
\Psi_{3}(\lambda)= & \tilde{A}_{1}(\lambda)^{T} P_{2}-P_{3}+S^{T} .
\end{aligned}
$$

Proof: Let

$$
\xi(t)=e^{\lambda t} x(t)
$$

Then, it is easy to see that the delay system $(\Sigma)$ is transformed to

$$
\begin{aligned}
& \dot{\xi}(t)=\tilde{A}(\lambda) \xi(t)+\tilde{A}_{1}(\lambda) \xi(t-h) \\
& \xi(t)=\phi(t)=e^{\lambda t} \varphi(t) \quad \forall t \in[-h, 0] .
\end{aligned}
$$

Under the condition of the theorem, we first show the asymptotic stability of the delay system (12). To this end, we define a Lyapunov functional candidate for (12) as follows:

$$
V\left(\xi_{t}\right)=V_{1}\left(\xi_{t}\right)+V_{2}\left(\xi_{t}\right)+V_{3}\left(\xi_{t}\right)
$$


where $t \geq h$, and

$$
\begin{aligned}
\xi_{t}= & \xi(t+\theta) \quad-2 h \leq \theta \leq 0 \\
V_{1}\left(\xi_{t}\right)= & \xi(t)^{T} P_{1} \xi(t) \\
V_{2}\left(\xi_{t}\right)= & 2 \xi(t)^{T} P_{2} \int_{t-h}^{t} \xi(\alpha) d \alpha \\
& +\left[\int_{t-h}^{t} \xi(\alpha) d \alpha\right]^{T} P_{3}\left[\int_{t-h}^{t} \xi(\alpha) d \alpha\right] \\
& +\int_{t-h}^{t} \xi(\alpha)^{T} Q \xi(\alpha) d \alpha \\
V_{3}\left(\xi_{t}\right)= & \int_{-h}^{0} \int_{t+\beta}^{t} \dot{\xi}(s)^{T} Z_{1} \dot{\xi}(s) d s d \beta \\
& +\int_{-h}^{0} \int_{t+\beta}^{t} \xi(s)^{T} Z_{2} \xi(s) d s d \beta .
\end{aligned}
$$

Then, we have the time derivative of $V_{i}\left(\xi_{t}\right), i=1,2,3$, along the trajectories of (12) as

$$
\begin{aligned}
\dot{V}_{1}\left(\xi_{t}\right)= & 2 \xi(t)^{T} P_{1}\left[\tilde{A}(\lambda) \xi(t)+\tilde{A}_{1}(\lambda) \xi(t-h)\right] \\
\dot{V}_{2}\left(\xi_{t}\right)= & 2\left[\tilde{A}(\lambda) \xi(t)+\tilde{A}_{1}(\lambda) \xi(t-h)\right]^{T} P_{2} \int_{t-h}^{t} \xi(\alpha) d \alpha \\
& +2 \xi(t)^{T} P_{2}[\xi(t)-\xi(t-h)] \\
& +2[\xi(t)-\xi(t-h)]^{T} P_{3} \int_{t-h}^{t} \xi(\alpha) d \alpha \\
& +\xi(t)^{T} Q \xi(t)-\xi(t-h)^{T} Q \xi(t-h) \\
\dot{V}_{3}\left(\xi_{t}\right)= & h \dot{\xi}(t)^{T} Z_{1} \dot{\xi}(t)+h \xi(t)^{T} Z_{2} \xi(t) \\
& -\int_{t-h}^{t} \dot{\xi}(\beta)^{T} Z_{1} \dot{\xi}(\beta) d \beta \\
& -\int_{t-h}^{t} \xi(\alpha)^{T} Z_{2} \xi(\alpha) d \alpha .
\end{aligned}
$$

By using the Newton-Leibniz formula

$$
\int_{t-h}^{t} \dot{\xi}(\beta) d \beta=\xi(t)-\xi(t-h)
$$

and (15)-(17), we obtain

$$
\begin{aligned}
\dot{V}\left(\xi_{t}\right)= & \xi(t)^{T}\left[P_{1} \tilde{A}(\lambda)+\tilde{A}(\lambda)^{T} P_{1}\right. \\
& \left.+P_{2}+P_{2}^{T}+Q+h Z_{2}\right] \xi(t) \\
& +2 \xi(t)^{T}\left[P_{1} \tilde{A}_{1}(\lambda)-P_{2}\right] \xi(t-h) \\
& +2 \xi(t)^{T}\left[\tilde{A}(\lambda)^{T} P_{2}+P_{3}\right] \int_{t-h}^{t} \xi(\alpha) d \alpha
\end{aligned}
$$

$$
\begin{aligned}
& +2 \xi(t-h)^{T}\left[\tilde{A}_{1}(\lambda)^{T} P_{2}-P_{3}\right] \int_{t-h}^{t} \xi(\alpha) d \alpha \\
& -\xi(t-h)^{T} Q \xi(t-h)+h[\tilde{A}(\lambda) \xi(t) \\
& \left.+\tilde{A}_{1}(\lambda) \xi(t-h)\right]^{T} Z_{1}\left[\tilde{A}(\lambda) \xi(t)+\tilde{A}_{1}(\lambda) \xi(t-h)\right]^{T} \\
& -\int_{t-h}^{t} \dot{\xi}(\beta)^{T} Z_{1} \dot{\xi}(\beta) d \beta-\int_{t-h}^{t} \xi(\alpha)^{T} Z_{2} \xi(\alpha) d \alpha \\
& +2 \xi(t)^{T} Y \int_{t-h}^{t} \dot{\xi}(\beta) d \beta-2 \xi(t)^{T} Y[\xi(t)-\xi(t-h)] \\
& +2 \xi(t-h)^{T} W \int_{t-h}^{t} \dot{\xi}(\beta) d \beta \\
& -2 \xi(t-h)^{T} W[\xi(t)-\xi(t-h)] \\
& +2 \int_{t-h}^{t} \xi(\alpha)^{T} d \alpha S \int_{t-h}^{t} \dot{\xi}(\beta) d \beta \\
& -2 \int_{t-h}^{t} \xi(\alpha)^{T} d \alpha S[\xi(t)-\xi(t-h)] \\
& =\frac{1}{h^{2}} \int_{t-h}^{t} \int_{t-h}^{t} \zeta(t, \alpha, \beta)^{T} \Gamma(\lambda) \zeta(t, \alpha, \beta) d \alpha d \beta
\end{aligned}
$$

where

$$
\begin{aligned}
& \zeta(t, \alpha, \beta)=\left[\begin{array}{llll}
\xi(t)^{T} & \xi(t-h)^{T} \quad \xi(\alpha)^{T} \quad \dot{\xi}(\beta)^{T}
\end{array}\right]^{T} \\
& \Gamma(\lambda)=\left[\begin{array}{cccc}
\Omega(\lambda) & \Psi_{1}(\lambda) & h \Psi_{2}(\lambda) & h Y \\
\Psi_{1}(\lambda)^{T} & -Q-W-W^{T} & h \Psi_{3}(\lambda) & h W \\
h \Psi_{2}(\lambda)^{T} & h \Psi_{3}(\lambda)^{T} & -h Z_{2} & h^{2} S \\
h Y^{T} & h W^{T} & h^{2} S^{T} & -h Z_{1}
\end{array}\right] \\
& +h\left[\begin{array}{c}
\tilde{A}(\lambda)^{T} Z_{1} \\
\tilde{A}_{1}(\lambda)^{T} Z_{1} \\
0 \\
0
\end{array}\right] Z_{1}^{-1}\left[\begin{array}{c}
\tilde{A}(\lambda)^{T} Z_{1} \\
\tilde{A}_{1}(\lambda)^{T} Z_{1} \\
0 \\
0
\end{array}\right]^{T}
\end{aligned}
$$

Now, by Schur complement, it follows from (3) that $\Gamma(\lambda)<0$. By this and (18), it is easy to have

$$
\dot{V}\left(\xi_{t}\right) \leq-a|\xi(t)|^{2}
$$

where $a=\lambda_{\min }(-\Gamma(\lambda))>0$. Now, let $k_{1}=$ $\max \left(\|\tilde{A}(\lambda)\|,\left\|\tilde{A}_{1}(\lambda)\right\|\right)$. Then, for any $t \geq 0$, it follows from (12) that

$$
\begin{aligned}
|\xi(t)| & =\left|\xi(0)+\int_{0}^{t}\left[\tilde{A}(\lambda) \xi(s)+\tilde{A}_{1}(\lambda) \xi(s-h)\right] d s\right| \\
& \leq|\xi(0)|+k_{1} \int_{0}^{t}[|\xi(s)|+|\xi(s-h)|] d s \\
& \leq|\xi(0)|+2 k_{1} \int_{-h}^{t}|\xi(s)| d s .
\end{aligned}
$$

$$
\left[\begin{array}{ccccc}
\Omega(\lambda) & \Psi_{1}(\lambda) & h \Psi_{2}(\lambda) & h Y & h \tilde{A}(\lambda)^{T} Z_{1} \\
\Psi_{1}(\lambda)^{T} & W+W^{T}-Q & h \Psi_{3}(\lambda) & h W & h \tilde{A}_{1}(\lambda)^{T} Z_{1} \\
h \Psi_{2}(\lambda)^{T} & h \Psi_{3}(\lambda)^{T} & -h Z_{2} & h^{2} S & 0 \\
h Y^{T} & h W^{T} & h^{2} S^{T} & -h Z_{1} & 0 \\
h Z_{1} \tilde{A}(\lambda) & h Z_{1} \tilde{A}_{1}(\lambda) & 0 & 0 & -h Z_{1}
\end{array}\right]<0
$$


Then, for any $0 \leq t \leq h$, we have

$$
\begin{aligned}
|\xi(t)| & \leq|\xi(0)|+2 k_{1} \int_{-h}^{0}|\xi(s)| d s+2 k_{1} \int_{0}^{t}|\xi(s)| d s \\
& \leq\left(2 k_{1} h+1\right)|\phi|_{h}+2 k_{1} \int_{0}^{t} \sup _{0 \leq r \leq s}|\xi(r)| d s .
\end{aligned}
$$

Therefore, for any $0 \leq t \leq h$,

$$
\sup _{0 \leq s \leq t}|\xi(s)| \leq\left(2 k_{1} h+1\right)|\phi|_{h}+2 k_{1} \int_{0}^{t} \sup _{0 \leq r \leq s}|\xi(r)| d s
$$

Applying the Gronwall-Bellman Lemma to this inequality gives that for any $0 \leq t \leq h$

$$
\sup _{0 \leq s \leq t}|\xi(s)| \leq\left(2 k_{1} h+1\right)|\phi|_{h} \exp \left(2 k_{1} h\right)
$$

Thus

$$
\sup _{0 \leq s \leq h}|\xi(s)|^{2} \leq\left(2 k_{1} h+1\right)^{2}|\phi|_{h}^{2} \exp \left(4 k_{1} h\right) .
$$

Note that

$$
\begin{aligned}
& \int_{-h}^{0} \int_{t+\beta}^{t} \dot{\xi}(s)^{T} Z_{1} \dot{\xi}(s) d s d \beta \leq h \int_{t-h}^{t} \dot{\xi}(s)^{T} Z_{1} \dot{\xi}(s) d s \\
& \int_{-h}^{0} \int_{t+\beta}^{t} \xi(s)^{T} Z_{2} \xi(s) d s d \beta \leq h \int_{t-h}^{t} \xi(s)^{T} Z_{2} \xi(s) d s .
\end{aligned}
$$

Then, by (14) and (21)-(23), we have

$$
\begin{aligned}
V\left(\xi_{h}\right) \leq & k_{2}\left[|\xi(h)|^{2}+\left(\int_{0}^{h} \xi(s) d s\right)^{2}\right. \\
& \left.+\int_{0}^{h}|\dot{\xi}(s)|^{2} d s+2 \int_{0}^{h}|\xi(s)|^{2} d s\right] \\
\leq & k_{2}\left(h^{2}+2 h+1\right) \sup _{0 \leq s \leq h}|\xi(s)|^{2} \\
& +k_{2} \int_{0}^{h}|\dot{\xi}(s)|^{2} d s \\
\leq & k_{2}(h+1)^{2} \sup _{0 \leq s \leq h}|\xi(s)|^{2} \\
& +k_{2} k_{1}^{2} \int_{0}^{h}[|\xi(s)|+|\xi(s-h)|]^{2} d s \\
\leq & k_{2}\left[(h+1)^{2}+2 h k_{1}^{2}\right] \sup _{0 \leq s \leq h}|\xi(s)|^{2} \\
& +2 k_{2} k_{1}^{2} h|\phi|_{h}^{2} \\
\leq & k_{3}|\phi|_{h}^{2}
\end{aligned}
$$

where

$$
\begin{aligned}
k_{2}= & \max \left(\lambda_{\max }(P), h \lambda_{\max }\left(Z_{1}\right), h \lambda_{\max }\left(Z_{2}\right), \lambda_{\max }(Q)\right) \\
k_{3}= & k_{2}\left[(h+1)^{2}+2 h k_{1}^{2}\right] \\
& \times\left(2 k_{1} h+1\right)^{2} \exp \left(4 k_{1} h\right)+2 k_{2} k_{1}^{2} h \\
P= & {\left[\begin{array}{ll}
P_{1} & P_{2} \\
P_{2}^{T} & P_{3}
\end{array}\right] . }
\end{aligned}
$$

Now, by (4), (14), and (19), it is easy to see that for any $t \geq h$

$$
\lambda_{\min }(P)|\xi(t)|^{2} \leq V\left(\xi_{t}\right) \leq V\left(\xi_{h}\right) .
$$

This together with (24) implies that for any $t \geq h$

$$
|\xi(t)|^{2} \leq \frac{k_{3}}{\lambda_{\min }(P)}|\phi|_{h}^{2}
$$

Noting the relationship in (11) and the inequality in (25), we have that for any $t \geq h$,

$$
|x(t)|^{2} \leq \frac{k_{3}}{\lambda_{\min }(P)} e^{-2 \lambda t}|\varphi|_{h}^{2}
$$

Similarly, by (20), we have

$$
\begin{aligned}
\sup _{0 \leq s \leq h}|x(s)|=\sup _{0 \leq s \leq h} e^{-\lambda s}|\xi(s)| & \\
& \leq\left(2 k_{1} h+1\right)|\varphi|_{h} \exp \left(2 k_{1} h\right) .
\end{aligned}
$$

Then, it follows from (26) and (27) that for any $t>0$

$$
\begin{aligned}
|x(t)| \leq \max \left(\left(2 k_{1} h\right.\right. & +1) \\
& \left.\times \exp \left(2 k_{1} h+\lambda h\right), \sqrt{\frac{k_{3}}{\lambda_{\min }(P)}}\right) e^{-\lambda t}|\varphi|_{h} .
\end{aligned}
$$

Therefore, by Definition 1, we have that the time-delay system $(\Sigma)$ is exponentially stable with a decay rate $\lambda$. This completes the proof.

Remark 1: Theorem 1 provides a new exponential stability condition for time-delay system $(\Sigma)$ in terms of LMIs. With this result, an upper bound of the decay rate can be calculated easily.

Remark 2: It is worth pointing out that the method in Theorem 1 can also be used to obtain exponential stability condition for neutral systems. To show this, we consider the following neutral system:

$$
\dot{x}(t)+D \dot{x}(t-h)=A x(t)+A_{1} x(t-h) .
$$

By (11), it is easy to see that the neutral system in (28) is transformed to

$$
\dot{\xi}(t)+\hat{D}(\lambda) \dot{\xi}(t-h)=\hat{A}(\lambda) \xi(t)+\hat{A}_{1}(\lambda) \xi(t-h)
$$

where

$$
\begin{aligned}
\hat{D}(\lambda) & =e^{\lambda h} D \quad \hat{A}(\lambda)=A+\lambda I \quad \hat{A}_{1}(\lambda) \\
& =e^{\lambda h}\left(A_{1}+\lambda D\right) .
\end{aligned}
$$

Choose a Lyapunov functional candidate for (28) as follows:

$$
V\left(\xi_{t}\right)=V_{1}\left(\xi_{t}\right)+V_{2}\left(\xi_{t}\right)+V_{3}\left(\xi_{t}\right)+V_{4}\left(\xi_{t}\right)
$$

where $V_{i}\left(\xi_{t}\right), i=1,2,3$, are given in (14), and

$$
V_{4}\left(\xi_{t}\right)=\int_{t-h}^{t} \dot{\xi}(s)^{T} Z_{3} \dot{\xi}(s) d s d \beta
$$


TABLE I

COMPARISON OF THE DECAY RATES IN EXAMPLE

\begin{tabular}{cccccccc}
\hline$h$ & 0.8 & 1 & 1.2 & 1.4 & 1.6 & 1.8 & 2.0 \\
\hline $\bar{\lambda}$ by Mondié and Kharitonov $[9]$ & 0.7344 & 0.6715 & 0.6145 & 0.5642 & 0.5202 & 0.4818 & 0.4481 \\
\hline $\bar{\lambda}$ by Liu et al. $[8]$ & 0.9367 & 0.5903 & 0.3400 & 0.1813 & 0.0752 & 0.0014 & 0 \\
\hline $\bar{\lambda}$ by Theorem 1 & 0.9366 & 0.9192 & 0.8991 & 0.8115 & 0.6990 & 0.6148 & 0.5494 \\
\hline
\end{tabular}

with $Z_{3}>0$. Then, following the same line as in the derivation of Theorem 1 , we can easily obtain a sufficient condition for exponential stability of the neutral system in (28).

By Theorem 1, it is easy to obtain the following delay-dependent asymptotic stability result for time-delay system $(\Sigma)$.

Corollary 1: The time-delay system $(\Sigma)$ is asymptotically stable if there exist matrices $P_{1}>0, P_{3}>0, Q>0, Z_{1}>0, Z_{2}>$ $0, Y, W, S$, and $P_{2}$ such that the following LMIs hold:

$$
\left.\begin{array}{ccccc}
\hat{\Omega} & \hat{\Psi}_{1} & h \hat{\Psi}_{2} & h Y & h A^{T} Z_{1} \\
\hat{\Psi}_{1}^{T} & W+W^{T}-Q & h \hat{\Psi}_{3} & h W & h A_{1}^{T} Z_{1} \\
h \hat{\Psi}_{2}^{T} & h \hat{\Psi}_{3}^{T} & -h Z_{2} & h^{2} S & 0 \\
h Y^{T} & h W^{T} & h^{2} S^{T} & -h Z_{1} & 0 \\
h Z_{1} A & h Z_{1} A_{1} & 0 & 0 & -h Z_{1}
\end{array}\right]<0
$$

where

$$
\begin{aligned}
\hat{\Omega}= & P_{1} A+A^{T} P_{1}+P_{2}+P_{2}^{T} \\
& -Y-Y^{T}+Q+h Z_{2} \\
\hat{\Psi}_{1}= & P_{1} A_{1}-P_{2}+Y-W^{T} \\
\hat{\Psi}_{2}= & A^{T} P_{2}+P_{3}-S^{T} \\
\hat{\Psi}_{3}= & A_{1}^{T} P_{2}-P_{3}+S^{T} .
\end{aligned}
$$

Remark 3: It is worth mentioning that although the LyapunovKrasovskii functional in (14) was also used in [14] to investigate the asymptotic stability of time-delay systems, the slack variable $S$ in Theorem 1 has not been introduced in [14] since in the derivation of asymptotic stability in [14] only single integrals were used while we use double integrals (see the proof of Theorem 1); the use of double integrals makes it possible to introduce the slack variable $S$ in our case. It is now well known that it is helpful to reduce conservatism in stability results for delay systems by introducing slack variables. Thus, the introduction of the slack variable $S$ in Corollary 1 may also reduce conservatism in the asymptotic stability condition in [14].

To show the reduced conservatism of the exponential stability condition in Theorem 1, we consider the time-delay system in [8] in the form of (1) with

$$
A=\left[\begin{array}{cc}
-3 & -2 \\
1 & 0
\end{array}\right] \quad A_{1}=\left[\begin{array}{cc}
-0.5 & 0.1 \\
0.3 & 0
\end{array}\right] \text {. }
$$

The upper bounds of the decay rate $\bar{\lambda}$ calculated by the methods in [8], [9] and Theorem 1 are compared in Table I. It can be seen that the result in Theorem 1 is less conservative than those in [8] and [9] for this example.

Now, we consider a time-delay system with time-varying normbounded parameter uncertainties described by

$$
\begin{aligned}
(\hat{\Sigma}): \quad \dot{x}(t)= & (A+\Delta A(t)) x(t) \\
& +\left(A_{1}+\Delta A_{1}(t)\right) x(t-h) \\
x(t)= & \phi(t) \quad \forall t \in[-h, 0]
\end{aligned}
$$

$$
\left[\begin{array}{cccccc}
\Omega(\lambda)+\epsilon E^{T} E & \Psi_{1}(\lambda)+\epsilon E^{T} E_{1} & h \Psi_{2}(\lambda) & h Y & h \tilde{A}(\lambda)^{T} Z_{1} & P_{1} D \\
\Psi_{1}(\lambda)^{T}+\epsilon E_{1}^{T} E & W+W^{T}-Q+\epsilon E_{1}^{T} E_{1} & h \Psi_{3}(\lambda) & h W & h \tilde{A}_{1}(\lambda)^{T} Z_{1} & 0 \\
h \Psi_{2}(\lambda)^{T} & h \Psi_{3}(\lambda)^{T} & -h Z_{2} & h^{2} S & 0 & h P_{2}^{T} D \\
h Y^{T} & h W^{T} & h^{2} S^{T} & -h Z_{1} & 0 & 0 \\
h Z_{1} \tilde{A}(\lambda) & h Z_{1} \tilde{A}_{1}(\lambda) & 0 & 0 & -h Z_{1} & h Z_{1} D \\
D^{T} P_{1} & 0 & h D^{T} P_{2} & 0 & h D^{T} Z_{1} & -\epsilon I
\end{array}\right]<0
$$

$\left[\begin{array}{cccccc}\hat{\Omega}+\epsilon E^{T} E & \hat{\Psi}_{1}+\epsilon E^{T} E_{1} & h \hat{\Psi}_{2} & h Y & h A^{T} Z_{1} & P_{1} D \\ \hat{\Psi}_{1}^{T}+\epsilon E_{1}^{T} E & W+W^{T}-Q+\epsilon E_{1}^{T} E_{1} & h \hat{\Psi}_{3} & h W & h A_{1}^{T} Z_{1} & 0 \\ h \hat{\Psi}_{2}^{T} & h \hat{\Psi}_{3}^{T} & -h Z_{2} & h^{2} S & 0 & h P_{2}^{T} D \\ h Y^{T} & h W^{T} & h^{2} S^{T} & -h Z_{1} & 0 & 0 \\ h Z_{1} A & h Z_{1} A_{1} & 0 & 0 & -h Z_{1} & h Z_{1} D \\ D^{T} P_{1} & 0 & h D^{T} P_{2} & 0 & h D^{T} Z_{1} & -\epsilon I\end{array}\right]<0$


TABLE II

COMPARISON OF THE DECAY RATES IN EXAMPLE 2

\begin{tabular}{cccccccc}
\hline$h$ & 0.3 & 0.5 & 0.7 & 0.9 & 1.1 & 1.3 & 1.5 \\
\hline $\bar{\lambda}$ by Mondié and Kharitonov $[9]$ & 0.6255 & 0.4760 & 0.3825 & 0.3191 & 0.2735 & 0.2392 & 0.2125 \\
\hline $\bar{\lambda}$ by Theorem 2 & 1.0108 & 0.8366 & 0.7103 & 0.6156 & 0.5425 & 0.4845 & 0.4375 \\
\hline
\end{tabular}

where

$$
\left[\Delta A(t) \quad \Delta A_{h}(t)\right]=D F(t)\left[\begin{array}{ll}
E & E_{1}
\end{array}\right]
$$

and $F(t) \in \mathbb{R}^{k \times l}$ is an unknown time-varying matrix function bounded by

$$
F(t)^{T} F(t) \leq I \quad \forall t
$$

By Theorem 1, it is easy to have the following result.

Theorem 2: For given scalars $\lambda>0$ and $h>0$, the uncertain time-delay system $(\hat{\Sigma})$ is robustly exponentially stable with a decay rate $\lambda$ if there exist matrices $P_{1}>0, P_{3}>0, Q>0, Z_{1}>0, Z_{2}>$ $0, Y, W, S, P_{2}$ and a scalar $\epsilon>0$ such that the first set of LMIs shown at the bottom of the previous page hold, where $\Omega(\lambda), \Psi_{1}(\lambda), \Psi_{2}(\lambda)$, and $\Psi_{3}(\lambda)$ are given in (7)-(10), respectively.

By Theorem 2, it is easy to have the following robust asymptotic stability results.

Corollary 2: The uncertain time-delay system $(\hat{\Sigma})$ is robustly asymptotically stable if there exist matrices $P_{1}>0, P_{3}>0, Q>$ $0, Z_{1}>0, Z_{2}>0, Y, W, S, P_{2}$ and a scalar $\epsilon>0$ such that the second set of LMIs shown at the bottom of the previous page hold, where $\hat{\Omega}, \hat{\Psi}_{1}, \hat{\Psi}_{2}$, and $\hat{\Psi}_{3}$ are given in (30)-(33), respectively.

To compare the robust exponential stability result in Theorem 2 with that in [9], we consider an uncertain time-delay system in the form of (34)-(37) with

$$
A=\left[\begin{array}{cc}
-4 & 1 \\
0 & -4
\end{array}\right] \quad A_{1}=\left[\begin{array}{cc}
0.1 & 0 \\
4 & 0.1
\end{array}\right]
$$

and $D=0.2 I, E=E_{1}=I$. Then, it is to see that this system can be rewritten in the form of that in [9, Ex. 2] with $\|\Delta A(t)\| \leq$ $0.2,\left\|\Delta A_{1}(t)\right\| \leq 0.2$. The comparison of the upper bound of the decay rates $\bar{\lambda}$ obtained by [9] and Theorem 2 is given in Table II, which shows that the condition in Theorem 2 is less conservative than that in [9] for this example.

\section{CONCLUSION}

This note has provided a new exponential stability condition for time-delay systems in terms of LMIs. Based on this, an upper bound of the decay rate can be calculated easily. When parameter uncertainties appear in a time-delay system, a new robust exponential stability condition has been proposed. Both the exponential stability and the robust exponential stability conditions proposed in this note are less conservative than some of those in the literature, which has been illustrated via two numerical examples.

\section{REFERENCES}

[1] J. Chen, "On computing the maximal delay intervals for stability of linear delay systems," IEEE Trans. Autom. Control, vol. 40, no. 6, pp. 1087-1093, Jun. 1995.
[2] E. Fridman and U. Shaked, "An improved stabilization method for linear time-delay systems," IEEE Trans. Autom. Control, vol. 47, no. 11, pp. 1931-1937, Nov. 2002.

[3] H. Gao and C. Wang, "Delay-dependent robust $H_{\infty}$ and $L_{2}-L_{\infty}$ filtering for a class of uncertain nonlinear time-delay systems," IEEE Trans. Autom. Control, vol. 48, no. 9, pp. 1661-1666, Sep. 2003.

[4] H. Gao and C. Wang, "A delay-dependent approach to robust $H_{\infty}$ filtering for uncertain discrete-time state-delayed systems," IEEE Trans. Signal Process., vol. 52, no. 6, pp. 1631-1640, Jun. 2004.

[5] A. Hmamed, "Comments on 'On an estimate of the decay rate for stable linear delay systems'," Int. J. Control, vol. 42, pp. 539-540, 1996.

[6] E. T. Jeung, J. H. Kim, and H. B. Park, " $H_{\infty}$-output feedback controller design for linear systems with time-varying delayed state," IEEE Trans. Autom. Control, vol. 43, no. 7, pp. 971-974, Jul. 1998.

[7] V. B. Kolmanovskii and A. D. Myshkis, Introduction to the Theory and Applications of Functional Differential Equations. Dordrecht, The Netherlands: Kluwer, 1999.

[8] P.-L. Liu, "Exponential stability for linear time-delay systems with delay dependence," J. Franklin Inst., vol. 340, pp. 481-488, 2003.

[9] S. Mondié and V. L. Kharitonov, "Exponential estimates for retarded time-delay systems: An LMI approach," IEEE Trans. Autom. Control, vol. 50, no. 2, pp. 268-273, Feb. 2005.

[10] T. Mori, N. Fukuma, and M. Kuwahara, "On an estimate of the decay rate for stable linear delay systems," Int. J. Control, vol. 36, pp. 95-97, 1982.

[11] S.-I. Niculescu, Delay Effects on Stability: A Robust Control Approach. Berlin, Germany: Springer-Verlag, 2001.

[12] S.-I. Niculescu, C. E. de Souza, L. Dugard, and J.-M. Dion, "Robust exponential stability of uncertain systems with time-varying delays," IEEE Trans. Autom. Control, vol. 43, no. 5, pp. 743-748, May 1998.

[13] S. S. Wang, B. S. Chen, and T. P. Lin, "Robust stability of uncertain time-delay systems," Int. J. Control, vol. 46, pp. 963-976, 1987.

[14] M. Wu, Y. He, and J.-H. She, "New delay-dependent stability criteria and stabilizing method for neutral systems," IEEE Trans. Autom. Control, vol. 49, no. 12, pp. 2266-2271, Dec. 2004.

[15] S. Xu and J. Lam, "Improved delay-dependent stability criteria for time-delay systems," IEEE Trans. Autom. Control, vol. 50, no. 3, pp. 384-387, Mar. 2005.

[16] S. Xu, J. Lam, and C. Yang, "Quadratic stability and stabilization of uncertain linear discrete-time systems with state delay," Syst. Control Lett., vol. 43, pp. 77-84, 2001. 